\title{
ARTICLE \\ ACSL4 is a predictive biomarker of sorafenib sensitivity in hepatocellular carcinoma
}

\author{
Ji Feng' ${ }^{1}$, Pei-zhi Lu', Guang-zhi Zhu' ${ }^{2,3,4}$, Shing Chung Hooi ${ }^{5}$, Yong Wu' ${ }^{1}$, Xiao-wei Huang ${ }^{1}$, Hui-qi Dai', Pan-hong Chen ${ }^{6}$, Zhong-jie Li' \\ Wen-jing Su' ${ }^{1}$, Chuang-ye Han ${ }^{2,3,4}$, Xin-ping Ye ${ }^{2,3,4}$, Tao Peng ${ }^{2,3,4}$, Jing Zhou ${ }^{5,6}$ and Guo-dong $\mathrm{Lu}^{1,3,7,8}$
}

\begin{abstract}
Sorafenib is the first-line treatment of advanced hepatocellular carcinoma (HCC). However, there is a lack of validated biomarkers to predict sorafenib sensitivity. In this study we investigated the role of ACSL4, a positive-activating enzyme of ferroptosis, in sorafenib-induced cell death and HCC patient outcome. We showed that ACSL4 protein expression was negatively associated with $I_{50}$ values of sorafenib in a panel of HCC cell lines $(R=-0.952, P<0.001)$. Knockdown of ACSL4 expression by specific siRNA/ sgRNA significantly attenuated sorafenib-induced lipid peroxidation and ferroptosis in Huh7 cells, and also rescued sorafenibinduced inhibition of xenograft tumor growth in vivo. We selected $29 \mathrm{HCC}$ patients with surgery as primary treatment and sorafenib as postoperative adjunct therapy from a hospital-based cohort. A high proportion $(66.7 \%)$ of HCC patients who had complete or partial responses to sorafenib treatment (according to the revised RECIST guideline) had higher ACSL4 expression in the pretreated HCC tissues, compared with those who had stable or progressed tumor growth $(23.5 \%, P=0.029)$. Since ACSL4 expression was independent of sorafenib treatment, it could serve as a useful predictive biomarker. Taken together, this study demonstrates that ACSL4 is essential for sorafenib-induced ferroptosis and useful for predicting sorafenib sensitivity in HCC. This study may have important translational impacts in precise treatment of HCC.
\end{abstract}

Keywords: hepatocellular carcinoma; ACSL4; sorafenib; ferroptosis; predictive biomarker

Acta Pharmacologica Sinica (2021) 42:160-170; https://doi.org/10.1038/s41401-020-0439-x

\section{INTRODUCTION}

Hepatocellular carcinoma ( $\mathrm{HCC}$ ) is the predominant primary malignancy in the liver. More importantly, it is the second leading cause of cancer death worldwide [1, 2]. Because of late diagnosis, a large proportion of HCC patients are diagnosed with disease in an intermediate (BCLC-B stage) or advanced stage (BCLC-C). Unfortunately, these two stages of $\mathrm{HCC}$ contraindicate the application of curative surgery. Even after curative surgery, approximately $50 \%$ of early-stage $\mathrm{HCC}$ patients recur within two years and progress into late-stage disease [3]. The median survival times of advanced and intermediate HCC patients are 6-8 and 16 months, respectively $[4,5]$. The first-line treatments for advanced HCC are two multikinase inhibitors, sorafenib and lenvatinib. However, two large, multicenter, randomized phase III clinical trials demonstrated that sorafenib extended survival by only two months $[6,7]$. Subsequent studies agreed, with sorafenib-treated advanced patients having a median survival time of 8.4-13.6 months and sorafenib-treated intermediate patients having a median survival time of 15.6-20.1 months
[8-10]. Although sorafenib is well tolerated, a proportion of HCC patients do not respond to sorafenib treatment. It is thus imperative to find a suitable biomarker to predict sorafenib sensitivity in HCC to achieve precise treatments that identify HCC patients who would benefit from sorafenib treatment and spare other potentially resistant patients from unnecessary severe side effects. However, after a decade of clinical and biomedical research into sorafenib, there is still a lack of validated predictive biomarkers in HCC [11].

The in vitro cytotoxicity of sorafenib has been well studied. It was reported that an iron-dependent type of programmed cell death, ferroptosis, rather than apoptosis is essential for sorafenibinduced cell death [12]. Ferroptosis has been implicated in the pathology of numerous diseases, including cancer, cardiovascular diseases (stroke, intracerebral hemorrhage, ischemia-reperfusion injury, etc.), and degenerative diseases (e.g., Parkinson's disease and Alzheimer's disease) [13-15]. Mechanistically, ferroptosis is executed through a series of enzymatic reactions that promote biosynthesis of polyunsaturated fatty acid (PUFA)-containing

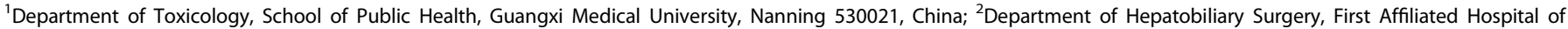

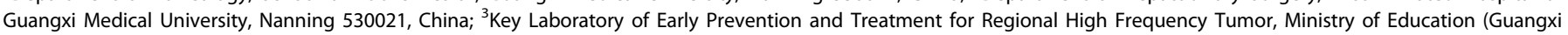

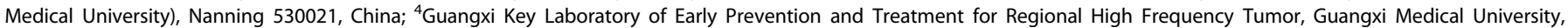

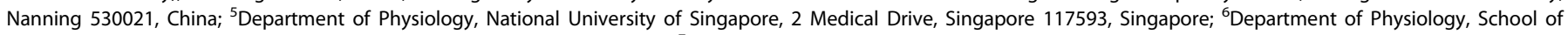

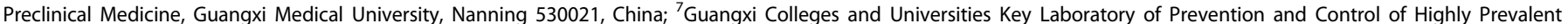

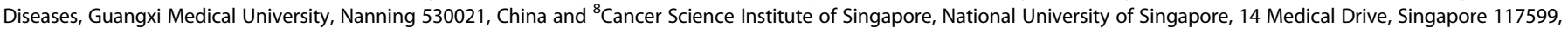
Singapore

Correspondence: Tao Peng (p98720p@163.com) or Jing Zhou (gardenia_zhou@hotmail.com) or Guo-dong Lu (golden_lu@hotmail.com)

The authors contributed equally: Ji Feng, Pei-zhi Lu, Guang-zhi Zhu

Received: 8 February 2020 Accepted: 12 May 2020

Published online: 15 June 2020 
ACSL4 predicts sorafenib sensitivity in HCC

J Feng et al.

phospholipids and iron-dependent accumulation of lipid peroxides. Two key ferroptosis-inducing signals, phosphatidylethanolamines (PEs) containing arachidonic acid (C20:4) and its elongation product adrenic acid (C22:4), are processed sequentially through ACSL4 (acyl-CoA synthetase long-chain family member 4) and lipoxygenase $[16,17]$. ACSL4 was shown to predict sensitivity to ferroptosis in a panel of basal-like breast cancer cell lines [16]. On the other hand, critical lipid peroxides are eradicated by glutathione peroxidase 4 (GPX4) and its cofactor glutathione (GSH) [13-15]. Ferroptosis can be induced by direct inhibition of GPX4 or indirect repression of SLC7A11 (also called System Xc-). SLC7A11 is a cystine-importing membrane protein that mediates GSH biosynthesis. It is noteworthy that sorafenib has been reported to block SLC7A11 function and GSH production, in addition to its well-known inhibition of many angiogenesis-associated kinases (VEGFR, FGFR, PDGFR, etc.) [18].

Thus, it is reasonable to speculate that genes involved in ferroptosis may regulate sorafenib sensitivity. A few studies have explored this hypothesis. Louandre and colleagues [19] studied the association of the retinoblastoma $(\mathrm{Rb})$ protein with sorafenibinduced ferroptosis. Their results demonstrated that $\mathrm{Rb}$ reduction could promote sorafenib-involved ferroptosis in HCC cells in vitro and in vivo, but they did not provide further mechanistic explanations. Later, Sun et al. [20] reported that nuclear factor erythroid 2-related factor 2 (NRF2) protected HCC cells from ferroptosis via transcriptional activation of ferritin heavy chain 1, heme oxygenase- 1 , and quinine oxidoreductase- 1 . As a negative feedback mechanism, sorafenib and other ferroptosis activators could activate NRF2 through p62 expression and Keap1 (Kelch-like $\mathrm{ECH}$-associated protein 1) inactivation. Two NRF2 transcripts, metallothionein-1 (MT-1) [21, 22] and Sigma-1 receptor [18], regulated redox accumulation and protected HCC cells from sorafenib-induced cell death. Furthermore, serum MT-1 expression was found to be increased in sorafenib-treated HCC patients, and this elevation was associated with relatively poor overall survival [21]. The above studies suggested the potential application of these genes as biomarkers for assessing sorafenib sensitivity. However, these genes can be altered by sorafenib treatment. In other words, the expression patterns of the studied genes are not a stable trait of HCC. It is thus difficult to predict sorafenib sensitivity before sorafenib treatment based on examinations of HCC biopsy or surgical tissue specimens.

In the present study, we determined the role of the positive-acting enzyme ACSL4 in sorafenib-induced ferroptosis in HCC cell lines and in surgical samples before sorafenib treatment. We first assessed the association between ACSL4 protein expression and the $\mathrm{IC}_{50}$ of sorafenib in a panel of HCC cell lines in vitro. Then, we demonstrated the dependency of ACSL4 expression on sorafenib-induced ferroptotic cell death in vitro and xenograft growth inhibition in vivo. Last, with a sorafenib-treated HCC cohort, we determined whether ACSL4 expression in HCC surgical tissue specimens could predict the outcome of subsequent sorafenib treatment.

\section{MATERIALS AND METHODS}

Human HCC samples and ethics

The study was approved by the University Institutional Review Board (\#20160302-10). The clinical data and tumor tissue samples were collected from an HCC cohort hosted by the Department of Hepatobiliary Surgery in the First Affiliated Hospital of Guangxi Medical University, China. All individuals who agreed to participate in the study signed consent forms. From the cohort, 29 HCC patients were selected based on two criteria: (1) radical resection as the primary treatment and sorafenib as the postoperative adjunct treatment; and (2) both HCC tissue samples and patient outcome data were available. All patients had undergone a standard three-month sorafenib treatment regimen before a thorough computed tomography (CT) examination and outcome

\begin{tabular}{|c|c|c|c|}
\hline Parameters & $\begin{array}{l}\text { Good-response } \\
\text { group }\end{array}$ & $\begin{array}{l}\text { Poor-response } \\
\text { group }\end{array}$ & $P$ value \\
\hline \multicolumn{4}{|l|}{ Sex } \\
\hline Male & 12 & 13 & 0.121 \\
\hline Female & 0 & 4 & \\
\hline Age $($ mean $\pm S D)$ & $50.7 \pm 8.4$ & $53.8 \pm 12.0$ & 0.441 \\
\hline HBV (\%) & 10 (83.3\%) & 14 (82.4\%) & 1.000 \\
\hline \multicolumn{4}{|l|}{$\mathrm{BCLC}$} \\
\hline A & 4 & 8 & 0.572 \\
\hline B & 5 & 4 & \\
\hline C & 3 & 5 & \\
\hline $\begin{array}{l}\text { AFP level after } \\
\text { surgery }(\mathrm{ng} / \mathrm{mL})\end{array}$ & $30.92 \pm 3.90$ & $198.56 \pm 22.98$ & 0.062 \\
\hline $\begin{array}{l}\text { AFP level during } \\
\text { CT scan (ng/mL) }\end{array}$ & $5.74 \pm 2.82$ & $427.38 \pm 28.73$ & $<0.001$ \\
\hline
\end{tabular}

evaluation. The posttreatment outcomes were evaluated according to the revised RECIST guidelines (version 1.1) [23]. The patients who had a complete response or partial response (at least $30 \%$ decreases in the sum of tumor diameters) after sorafenib treatment were regarded as having a good outcome $(n=12)$. In contrast, those who had stable tumors or even progressive tumor growth (at least $20 \%$ increases in the sum of tumor diameters) or severe side effects were regarded as having a poor outcome $(n=$ 17). The classification of the patients into the two groups was verified by two senior doctors. The clinicopathological characteristics of the two groups are listed in Table 1.

Reagents and antibodies

Sorafenib was purchased from Target Mol (Shanghai, China); inhibitors including RSL-3, erastin, ferrostatin-1, liproxstatin-1, necrostatin-1, deferoxamine mesylate, z-VAD-FMK and pioglitazone were obtained from MedChemExpress (Shanghai, China); 2,4thiazolidinedione was obtained from Abcam (Shanghai, China); rosiglitazone, L-buthionine-sulfoximine (BSO), GSH monoethyl ester (GSH-MEE) and other chemicals not indicated elsewhere were obtained from Sigma-Aldrich (Shanghai, China). Primary antibodies against ACSL3 (\#sc-271246), ACSL4 (\#sC-271800), ACSL5 (\#sC-100473) and ACSS2 (\#sC-85258) were purchased from Santa Cruz Biotechnology (Dallas, Texas, USA); antibodies against GPX4 (\#ab125066) and ACSL4 (\#ab155282) were purchased from Abcam; an anti-ACSL1 antibody (\#9189) was purchased from Cell Signaling Technology (Beverly, MA, USA); and an anti-a-tubulin antibody (\#T-5168) was purchased from Sigma-Aldrich.

Cell culture

The HCC cell lines PLC/5, SNU-182, SNU-387, SNU-449, SNU-398, and HepG2 were obtained from American Type Culture Collection. Huh7 cell line was obtained from the Shanghai Institute of Biochemistry and Cell Biology (Shanghai, China) while HCC-M was kindly provided by Dr E.C. Ren (National University of Singapore). These cells were cultured in either RPMI-1640 medium or Dulbecco's modified Eagle's medium supplemented with $10 \%$ fetal bovine serum (Gibco, Newcastle, NSW, Australia) and $100 \mathrm{U} / \mathrm{mL}$ penicillin and streptomycin in a $5 \% \mathrm{CO}_{2}$ incubator at $37^{\circ} \mathrm{C}$.

Determination of cell viability and cell death

Cell viability was determined with an MTT assay (Amersco, Houston, Texas, USA) according to the manufacturer's instructions. 
A colony formation assay was used to monitor long-term cell growth as described previously [24]. Additionally, cell death was evaluated with a PI (propidium iodide) exclusion assay as described previously [25]. PI-positive cells with impaired cell membranes were regarded as dead cells and assessed by flow cytometry (CytoFLEX FCM, Beckman, Atlanta, Georgia, USA).

\section{Western blot analysis}

Western blotting was used to analyze protein expression as described previously [26]. In brief, equal amounts of protein extracts were separated on SDS-PAGE gels and transferred to PVDF membranes (Bio-Rad, Shanghai, China). After incubation with corresponding antibodies, signals were visualized by enhanced chemiluminescence (Thermo Fisher, Shanghai, China) according to the manufacturer's instructions. Semiquantitative analysis was performed with ImageJ software ( $\mathrm{NIH}$, https://imagej. nih.gov/ij/download.html) [27].

\section{ACSL4 silencing with siRNA or a CRISPR/Cas9 system and transient overexpression}

Synthetic stealth small interfering RNAs [siRNAs; a scrambled siRNA and an ACSL4-targeting siRNA (\#HSS103537 or \#HSS103539)] were purchased from Invitrogen (Shanghai, China), while CRISPR/Cas9 dualvector lentiviral particles containing single guide RNAs (sgRNAs; a universal negative control sgRNA and an ACSL4-targeting sgRNA)] were purchased from GeneChem (Shanghai, China). The siRNAs were transfected with Lipofectamine RNAiMAX (Invitrogen) according to the manufacturer's instructions. Lentivirus-transduced cells were selected for stable knockdown of ACSL4 with $2 \mu \mathrm{g} / \mathrm{mL}$ puromycin (Sigma-Aldrich). The sgRNA sequences are listed below: NC-sg (CGC TTC CGC GGC CCG TTC AA); and ACSL4-sg1 (TCA TGG GCT AAA TGA ATC TG); ACSL4-sg2 (CTG TGA ATC TCA AAT CCT TC). The human ACSL4 gene (NM_001318509.2) was cloned into a pCMV-Tag2B plasmid. Then, transient overexpression of ACSL4 or an empty vector was established by transduction as described above with the aid of Lipofectamine 2000 [26].

Immunohistochemical staining

ACSL4 protein expression was determined by immunohistochemistry (IHC) performed as described previously [26]. Briefly, human HCC tissue sections were incubated with an anti-ACSL4 antibody (\#ab155282, Abcam) overnight at $4{ }^{\circ} \mathrm{C}$, followed by processing with a standard biotin-streptavidin HRP detection system (Zsbio, Beijing, China) according to the manufacturer's instructions. The tissue sections were then counterstained with hematoxylin, dehydrated and mounted. In addition, xenograft tissue samples collected from nude mice were stained with an anti-ACSL4 antibody (\#ab155282, Abcam) and anti-Ki67 antibody (\#ab92742, Abcam).

Determination of reactive oxygen species and lipid peroxidation levels

Cellular levels of reactive oxygen species (ROS) were determined with CM-H2DCFDA (Invitrogen) as previously described [26]. The relative concentrations of malondialdehyde (MDA) and GSH in cell lysates were assessed by the use of appropriate biochemical assay kits (BioVision, Milpitas, CA, USA) according to the manufacturer's instructions. The level of lipid peroxidation was measured with Bodipy 581/591 C11 (Invitrogen). Briefly, after resuspension, cells were incubated with $2 \mu \mathrm{M}$ Bodipy probe for $15 \mathrm{~min}$ at room temperature. Fluorescence was then measured at 595 and $510 \mathrm{~nm}$ by a multimode microplate reader (SPARK $10 \mathrm{M}$, Tecan, Morrisville, NC, USA). The ratio of the 510/595 fluorescence intensities was calculated to analyze the extent of lipid peroxidation.

\section{HCC xenograft experiment}

The nude mouse xenograft experiment was approved by the Guangxi Medical University Institutional Animal Care and Use Committees and performed in accordance with the Association for Assessment and
Accreditation of Laboratory Animal Care guidelines. A total of 32 male Balb/c nude mice (6-8 weeks old) were obtained from Hunan SJA Laboratory Animal Co., Ltd. (Changsha, China) and then separated randomly into 4 groups. Five million control or stable ACSL4-silenced Huh7 cells were inoculated subcutaneously into the right flank of the nude mice. Once the inoculated tumor size reached $20-50 \mathrm{~mm}^{3}$, the mice were given PBS or sorafenib $(30 \mathrm{mg} / \mathrm{kg}$, once every other day) by gavage. Tumors were measured every day with calipers, and tumor volume was calculated using the formula (length $\times$ width $\times$ width)/2. On the 12th day after treatment, all mice were sacrificed, and the tumor nodules were collected for subsequent experiments.

Statistical analyses

Experimental differences were determined with the SPSS software package (version 22.0, IBM SPSS Statistics for Windows, Chicago, IL, USA) using ANOVA LSD, two-sided Student's $t$ test or Fisher's exact test analysis. Quantitative data are expressed as the mean \pm SD of at least three independent experiments. A $P$ value $<0.05$ was considered statistically significant.

\section{RESULTS}

ACSL4 expression predicted sorafenib sensitivity in a panel of HCC cell lines

Sorafenib-induced ferroptosis was confirmed based on the following experimental results. Neither the apoptosis inhibitor zVAD-FMK nor the necroptosis inhibitor necrostatin-1 could prevent sorafenib-induced cell death in Huh7 cells (Fig. 1a). In contrast, cell death could be repressed by cotreatment with four different ferroptosis inhibitors (Fig. 1b). Among them, liproxstatin1 and ferrostatin-1 block lipid peroxidation, while GSH-MEE is a membrane-permeable GSH derivative that restores the GSH supply, and deferoxamine depletes iron. Consistent with the repressed cell death results, these inhibitors also protected cells from sorafenib-induced lipid peroxidation (Fig. 1c), as measured by the fluorescence shift of the Bodipy 581/591 C11 dye from 595 to $510 \mathrm{~nm}$.

We then determined the correlation of ACSL4 protein expression with sorafenib sensitivity in a panel of HCC cell lines. We first measured the protein expression of ACSL4 together with that of other acyl-CoA synthetase long-chain family members. As shown in Fig. 1d, ACSL4 expression was high in Huh7 and PLC/5 cells, deficient in SNU-182 cells, and low in the other cell lines. Second, we determined the $\mathrm{IC}_{50}$ of sorafenib required to induce growth inhibition (Fig. 1e). Huh7 cells, which have high ACSL4 expression, had the lowest IC $\mathrm{C}_{50}(1.8 \mu \mathrm{M})$, while ACSL4-deficient SNU-182 cells had an $\mathrm{IC}_{50}$ greater than $16 \mu \mathrm{M}$. More importantly, the protein expression of ACSL4 but not that of the other ACSL family members was negatively associated with the $\mathrm{IC}_{50}$ of sorafenib $(R=-0.952, P<0.001$, Fig. If and Supplementary Fig. 1). Notably, unlike another essential protein (GPX4), ACSL4 expression was stable (dose and time) independent of sorafenib treatment (Fig. 1g, h). In stable control Huh7 cells transfected with a scramble sgRNA, sorafenib treatment reduced GPX4 expression (Supplementary Fig. 1c). However, in stable cells with ACSL4 knocked out, GPX4 protein expression was upregulated by sorafenib. Thus, the above results demonstrated that ACSL4 could act as a stable biomarker for assessing sorafenib sensitivity in HCC cell lines in vitro.

Transient silencing of ACSL4 protected cells from sorafenibinduced ferroptosis

To determine the dependency on ACSL4 in sorafenib-induced cell death, we transiently knocked down ACSL4 with specific siRNAs. As shown in Fig. 2a, ACSL4-silenced Huh7 cells became resistant to sorafenib treatment. In line with this, cotreatment with one of three reported ACSL4 inhibitors, rosiglitazone [28], pioglitazone [29] or 2,4-thiazolidinedione (2,4-TZD) [16], reduced sorafenib- 

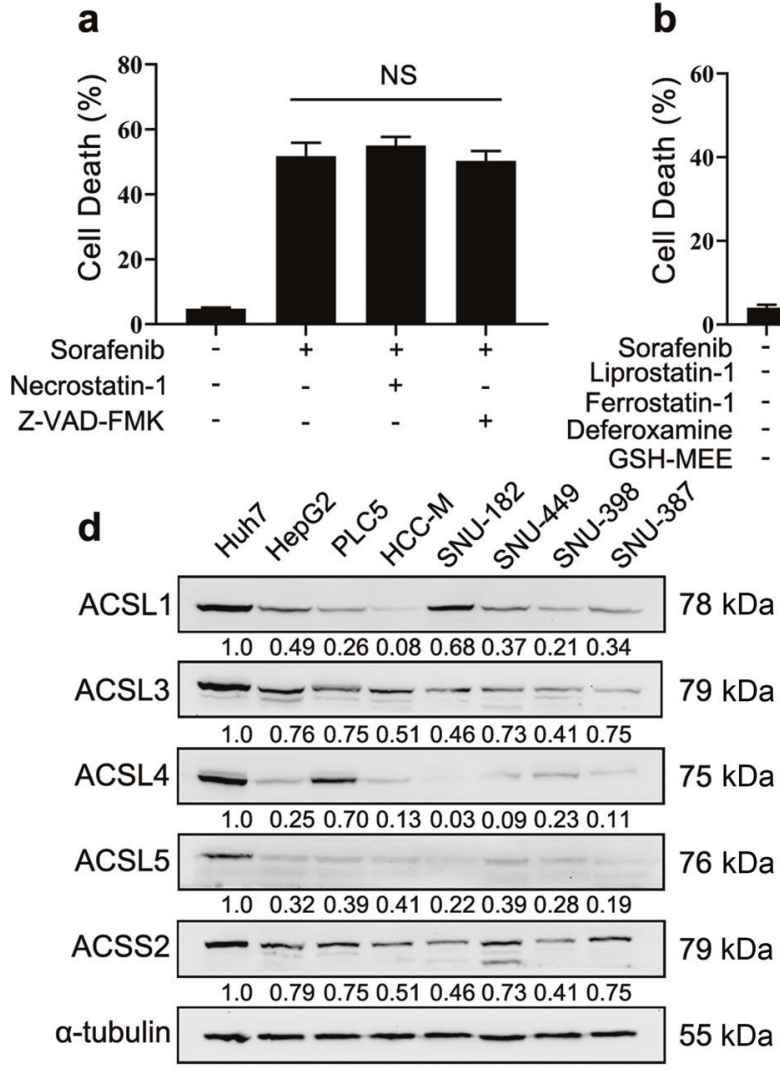

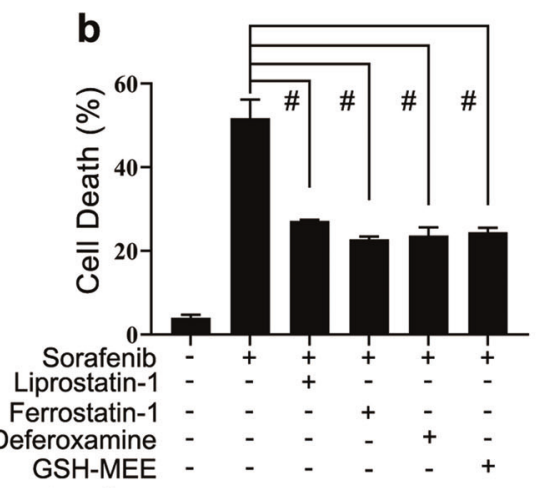

c

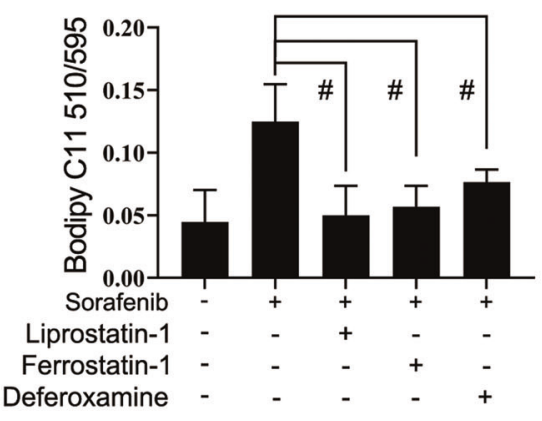

e

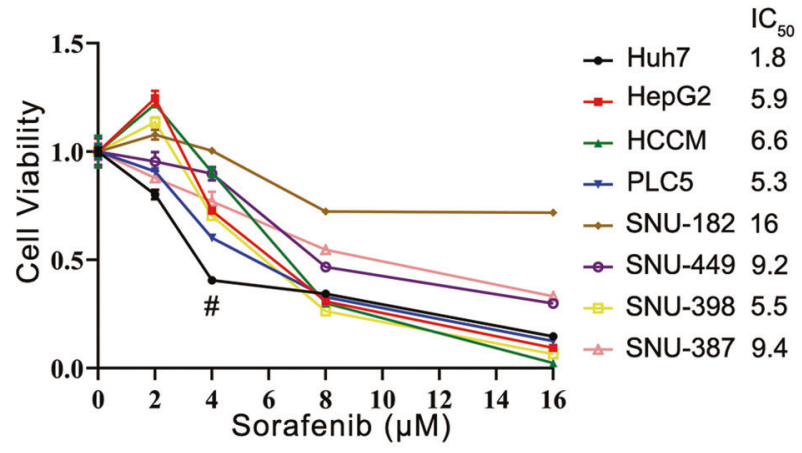

g

h
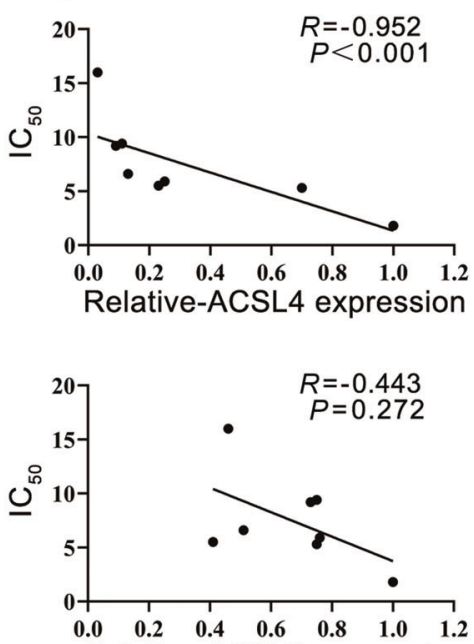

Relative-ACSL3 expression
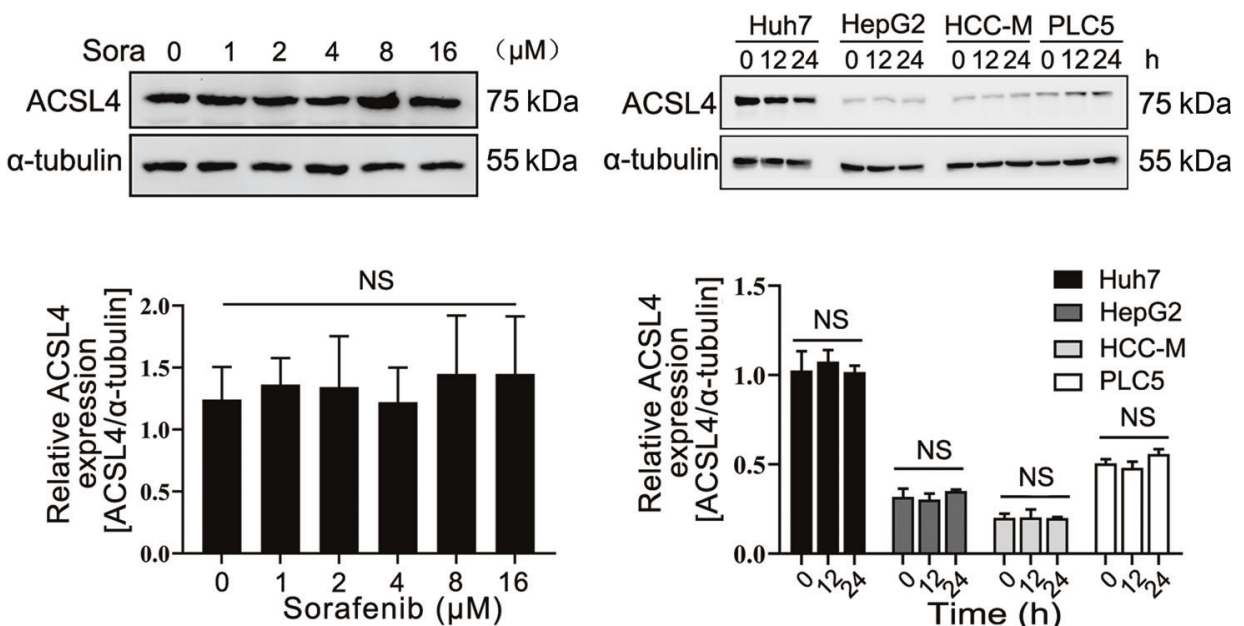

Fig. 1 ACSL4 expression was positively associated with sorafenib sensitivity in HCC cell lines. a Huh7 cells were treated with sorafenib $(10 \mu \mathrm{M})$ with or without the apoptosis inhibitor z-VAD-FMK $(50 \mu \mathrm{M})$ or necroptosis inhibitor necrostatin-1 (50 $\mu \mathrm{M})$ for $24 \mathrm{~h}$. b Huh7 cells were treated with sorafenib with or without a ferroptosis inhibitor $(2 \mu \mathrm{M}$ ferrostatin-1, $200 \mathrm{nM}$ liprostatin-1, $200 \mu \mathrm{M}$ deferoxamine or $5 \mathrm{mM}$ GSHMEE) for $24 \mathrm{~h}$. c Huh7 cells were treated as in (b) for $6 \mathrm{~h}$ before examination of lipid peroxidation with Bodipy 581/591 C11. d Western blot analysis of ACSL4 and other ACSL family members in the indicated HCC cell lines was performed. e Different HCC cell lines were treated with increasing doses of sorafenib for $24 \mathrm{~h}$, and then the corresponding $\mathrm{IC}_{50} \mathrm{~s}$ were determined from the dose-response curves and are shown on the right. $f$ The sorafenib IC 50 was correlated with ACSL4 (upper panel) or ACSL3 expression (lower panel) by Spearman's rank correlation analysis. $\mathbf{g}$, $\mathbf{h}$ HCC cells were treated with sorafenib as indicated. ACSL4 expression was monitored by Western blotting, and its expression relative to that of $a$-tubulin is shown in the lower panels. NS not significant, ${ }^{\#} P<0.05$.

induced cell death (Fig. 2b). We then validated the role of ACSL4 in ferroptosis by examining redox species. ACSL4 silencing consistently repressed the sorafenib-induced production of reactive oxygen species as determined with DCFDA (Fig. 2c), lipid peroxidation as determined with Bodipy 581/591 C11 (Fig. 2d), and malondialdehyde (MDA, one of the final products of PUFA peroxidation) as determined with biochemical assays (Fig. 2e). In contrast, ACSL4 silencing restored the sorafenib-induced GSH reduction (Fig. 2f). In low-expressing HepG2 cells, enforced overexpression of ACSL4 consistently increased cellular sensitivity 
a

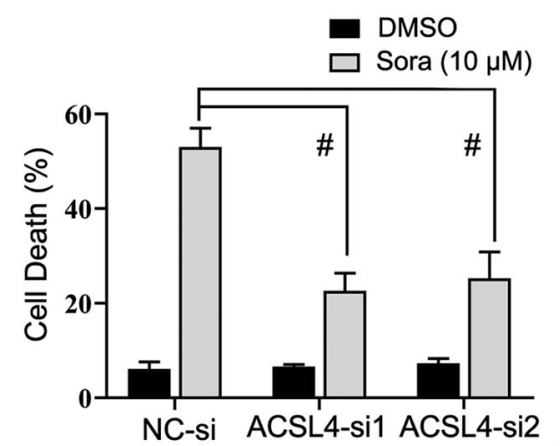

C

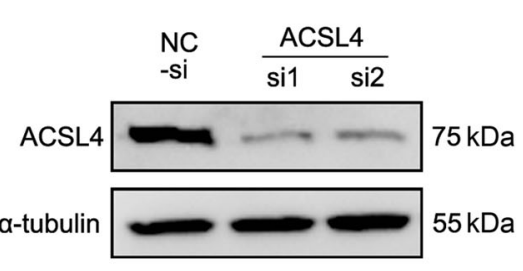

b
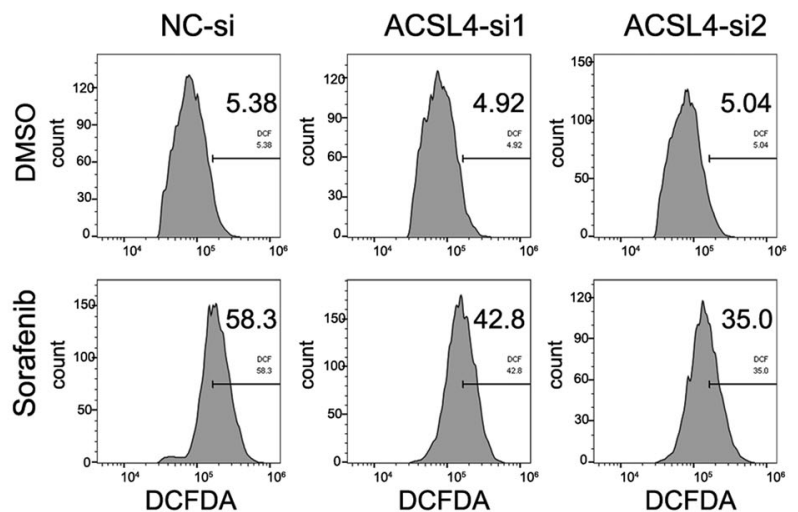

2,4-Thiazolidinedione -
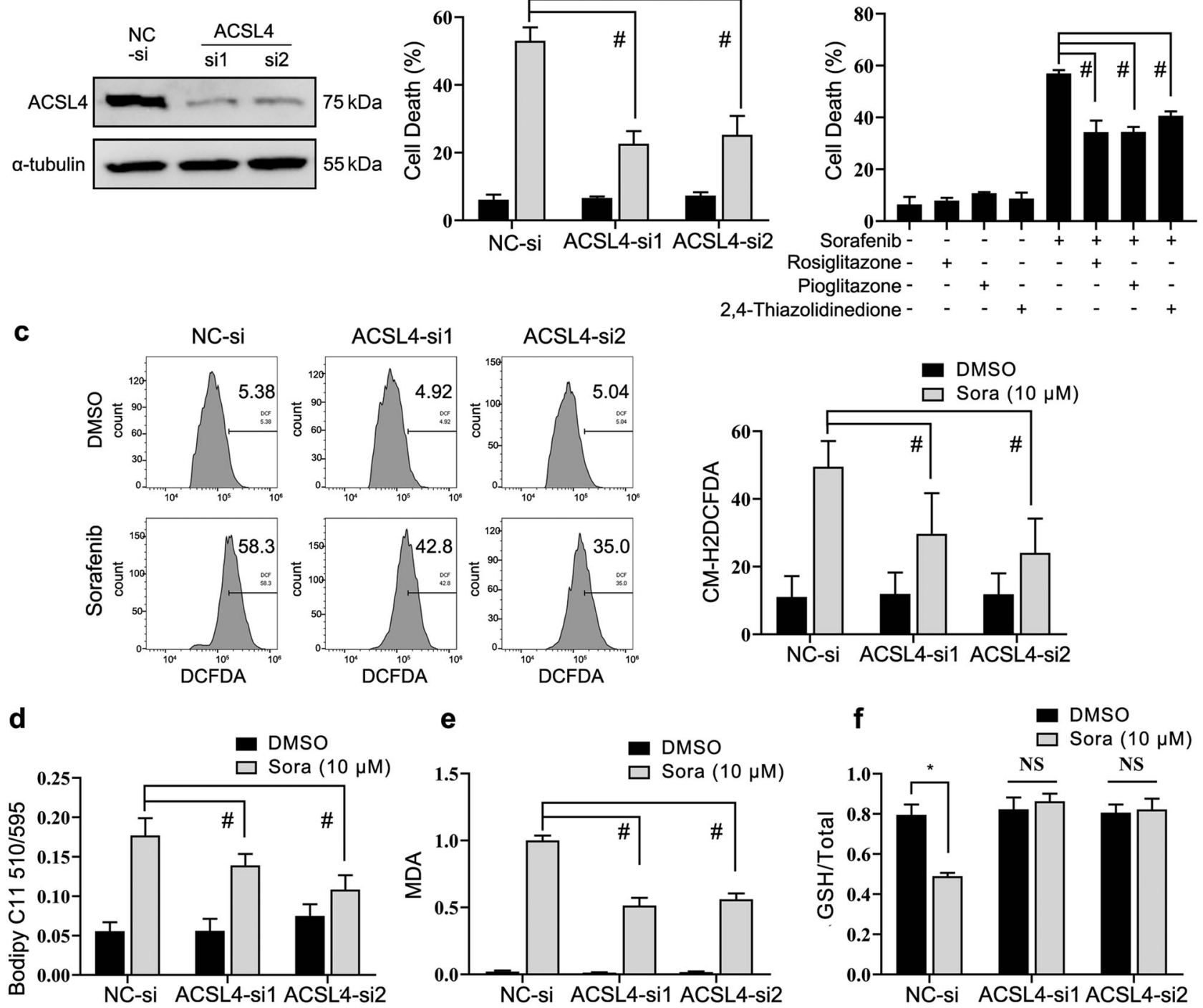

g
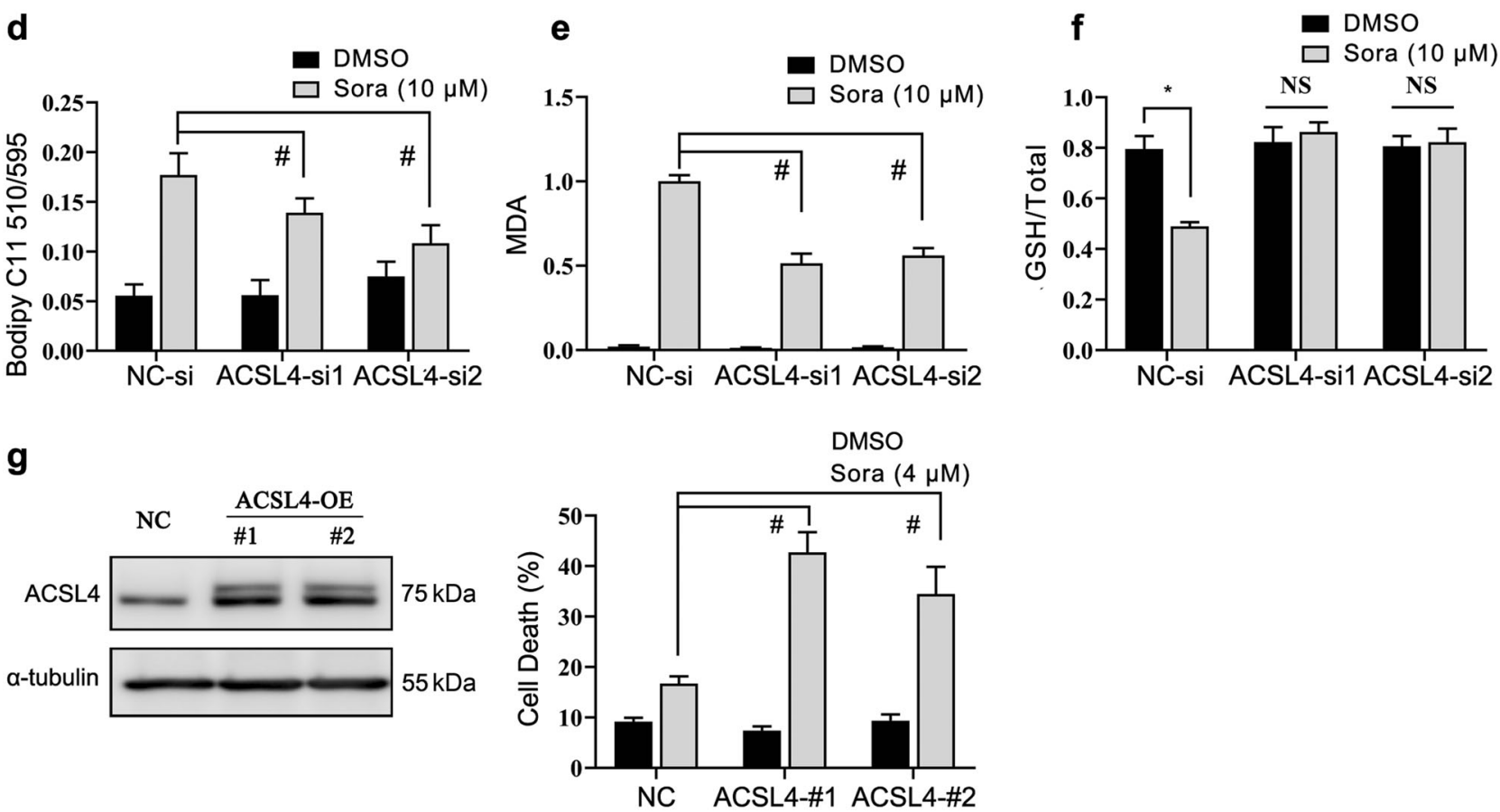

Fig. 2 Transient silencing of ACSL4 protected sorafenib-induced ferroptosis. a Huh7 cells were transfected with a scramble control siRNA (NC-si) or specific siRNAs targeting ACSL4 (ACSL4-si1 and ACSL4-si2). Cell death after sorafenib treatment was determined by a PI exclusion assay. b Huh7 cells were treated with sorafenib $(10 \mu \mathrm{M})$ with or without an ACSL4 inhibitor ( $20 \mu \mathrm{M}$ rosiglitazone, $20 \mu \mathrm{M}$ pioglitazone, or $20 \mu \mathrm{M}$ 2,4-thiazolidinedione) for $24 \mathrm{~h}$. c After siRNA transfection, Huh7 cells were treated with sorafenib for $12 \mathrm{~h}$ before examination of reactive oxygen species with CM-H2DCFDA. The quantification bar chart is shown on the right. d-f After the indicated sorafenib treatment as in (c), the changes in lipid peroxidation (d), MDA (e) and GSH content (f) were determined with the appropriate biochemical assays. $\mathbf{g}$ ACSL4 was overexpressed in HepG2 cells with low endogenous ACSL4 expression. The resultant changes in cell death were determined after sorafenib treatment as shown in (a). 
to sorafenib treatment (Fig. 2g). Thus, sorafenib-induced ferroptosis is dependent on ACSL4 activity.

Stable knockout of ACSL4 protected cells from ferroptosis induced by different ferroptosis activators

To further validate the role of ACSL4 and to facilitate the investigation of xenograft tumor growth, we performed stable knockout of ACSL4 using a CRISPR/Cas9 system with specific sgRNAs. Stable ACSL4 knockout did not affect cell proliferation rates (Fig. 3a). The results consistently showed that cells stably deficient in ACSL4 were resistant to sorafenib-induced cell death (Fig. 3b), MDA production (Fig. 3c) and GSH reduction (Fig. 3d). The protective effects of ACSL 4 knockout were reproducible in treatments with other ferroptosis activators including erastin, RSL3 and BSO (Fig. 3e). Next, we tested the long-term effect of ACSL4 knockout with a colony formation assay. Stable knockout of ACSL4 alone did not affect cell growth or the number of colonies. Importantly, ACSL4 knockout markedly rescued the sorafenibinduced inhibition of colony growth (Fig. 3f).

\section{ACSL4 knockout rescued HCC xenograft growth after sorafenib treatment}

We then inoculated stable ACSL4-knockout or control cells into the right flank of nude mice. Similar to the results of the colony formation assay, ACSL4 knockout alone did not alter the ability of HCC cells to form xenograft nodules (Fig. 4a). The size of the tumor nodules developed from stable control cells was the same as that of the nodules developed from stable ACSL4-knockout cells.

Then, the tumor-bearing mice were given oral sorafenib treatment $(30 \mathrm{mg} / \mathrm{kg}$ mouse weight, every other day) or the same volume of PBS using the gavage technique. Sorafenib significantly repressed xenograft tumor growth in the mice inoculated with stable scramble control sgRNA-transfected cells (Fig. 4a, b). Body weight did not differ among different groups after sorafenib or PBS treatment (Fig. 4c). Histopathological examination found that sorafenib induced extensive cell death $(56 \%$ of the area in tissue sections, as shown by a smeared cell morphology and loss of nuclear staining) in tumor nodules (Fig. $4 \mathrm{~d}$ and Supplementary Fig. 2). In contrast, the same treatment failed to inhibit tumor growth in mice inoculated with stable ACSL4-knockout cells. The resulting tumor nodules were also less affected by cell death. Furthermore, we performed $\mathrm{IHC}$ for $\mathrm{Ki}-67$ to monitor the cell proliferation status. The suppressive effect of sorafenib on Ki-67 staining positivity was observed only in the tumor nodules developed from the stable control cells, not in those nodules developed from stable ACSL4-knockout cells (Fig. 4e). More importantly, ACSL4 expression was stable after sorafenib treatment in vivo (Fig. $4 \mathrm{e}, \mathrm{f}$ ), which is consistent with the cellular experiment results (Fig. 1g, h). Finally, MDA content was significantly increased in the control nodules but not in the ACSL4-knockout nodules after sorafenib treatment (Fig. 4g), strengthening the evidence for the essential roles of ACSL4 in sorafenib-induced lipid peroxidation and ferroptosis in vivo. Therefore, the above results collectively demonstrated that ACSL4 was essential for sorafenib-induced ferroptotic cell death in HCC in vivo.

High ACSL4 expression in HCC tissue was associated with relatively good patient outcomes after sorafenib treatment To assess whether ACSL4 expression in HCC clinical samples is useful for predicting sorafenib sensitivity, we analyzed our hospital-based HCC cohort. HCC tissue sections and corresponding clinical data were collected from primary HCC patients who underwent surgery as their primary treatment and subsequent systemic sorafenib as their postoperative adjunct treatment. First, both Western blot analysis (Fig. 5a) and IHC (Fig. 5b, c) confirmed that the ACSL4 protein was overexpressed in HCC tissue $(n=29)$ compared with neighboring nontumor liver tissue $(n=21, P=$ 0.000118). Second, bioinformatic analysis using a GEO dataset
(GSE14520) showed that ACSL4 was the most significantly upregulated gene in HCC tissue specimens among all ACSL family members (fold change $=9.85, P=1.26 \mathrm{E}-81$, Supplementary Fig. 4a). In contrast, the mRNA levels of ACSL1 and ACSL5 were downregulated, while that of ACSL3 was moderately upregulated. Similar analyses using the TCGA-LIHC dataset reproducibly revealed that ACSL4 expression was upregulated in HCC tissue compared to adjacent normal liver tissue (fold change $=4.535$, $P=8.71$ E-16, Supplementary Fig. 4b).

Last, we compared the pretreatment ACSL4 protein expression in HCC tissue samples between patients with a good response and those with a poor response after sorafenib treatment. Representative IHC images of ACSL4 from good- and poor-response patients are shown in Fig. $5 \mathrm{~d}$. The cutoff level to differentiate high and low expression of ACSL4 in HCC tissue samples was calculated based on an AUROC curve analysis (data not shown) and the $95 \%$ upper percentile of ACSL4 expression in nontumor liver tissue specimens. As shown in Table $2 \mathrm{a}$, among the HCC patients with a good response after sorafenib treatment, $66.7 \%$ (8 out of 12 ) had high ACSL4 expression ( $\geq 0.4$ AOD) in their pretreatment HCC tissue. In contrast, only $23.5 \%$ (4 out of 17 ) of the HCC patients with a poor prognosis had high ACSL4 expression $(P=0.02)$. Notably, the good-response patients had decreased AFP levels after sorafenib treatment (from $30.92 \pm 3.90$ to $5.74 \pm 2.82 \mathrm{ng} / \mathrm{mL}$ ), while the poor-response patients had increased AFP levels (from $198.56 \pm 22.98$ to $427.38 \pm 28.73 \mathrm{ng} / \mathrm{mL}$, Table 1). To further exclude a possible bias in patient selection, we included only $\mathrm{HCC}$ patients with an AFP level less than $200 \mathrm{ng} / \mathrm{mL}$ after surgery $(30.92 \pm 3.90 \mathrm{ng} / \mathrm{mL}$ for the good-response patients and $19.82 \pm$ 4.39 for the poor-response patients) and redid the analysis. The results consistently showed that a higher proportion of the goodresponse patients than the poor-response patients had high ACSL4 expression ( $66.7 \%$ vs. $20.0 \%, P=0.043$, Table 2b). Taken together, these results may suggest that high ACSL4 expression in HCC tissue is associated with better patient responses to subsequent sorafenib treatment.

\section{DISCUSSION}

The present study demonstrated that ACSL4 protein expression can predict sorafenib sensitivity in a panel of HCC cell lines in vitro. More importantly, high ACSL4 protein expression in HCC clinical tissue specimens was associated with a good patient outcome after subsequent sorafenib treatment. ACSL4 inhibition by both genetic approaches (silencing/knockout using a specific siRNA or CRISPR/Cas9 sgRNA) and pharmacological approaches could rescue sorafenib-induced ferroptotic cell death in vitro and xenograft growth inhibition in vivo.

Ferroptosis is characterized by iron-dependent lipid peroxidation, which is distinct from apoptosis, necrosis and other types of cell death. However, not all kinds of lipid oxidative species contribute to ferroptosis. Based on a quantitative redox lipidomic approach, Kagan and colleagues found that only oxidized arachidonic and adrenic PEs were critical for ferroptosis execution [17]. Another study used CRISPR/Cas9-based genetic screening approaches and demonstrated that ACSL4 is the essential enzyme for PE conversion and ferroptosis induction [16]. Furthermore, the latter study found that ACSL4 expression could predict ferroptosis sensitivity in basal-like breast cancer cell lines. Our current study supported the usefulness of ACSL4 expression as a predictive biomarker for ferroptosis sensitivity in different HCC cell lines in vitro (Fig. 1) and in clinical HCC patients (Table 1). The protein expression of ACSL4 was negatively associated with the sorafenib $I_{50}$ (Fig. 1). The dependency of ACSL4 expression on sorafenibinduced lipid peroxidation and ferroptosis was then confirmed by ACSL4 silencing/knockout experiments (Figs. 2 and 3). ACSL4 knockout also rescued sorafenib-induced inhibition of xenograft tumor growth (Fig. 4). More importantly, high ACSL4 expression in 
a
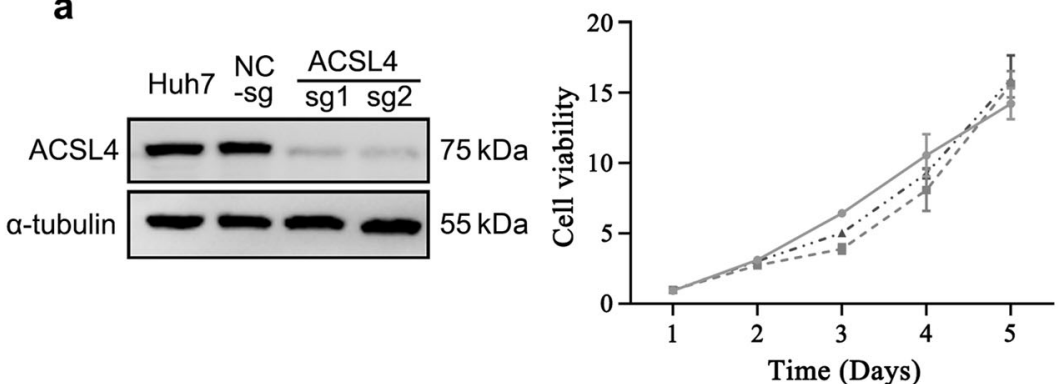

$\rightarrow$ NC-sg

- - ACSL4-sg1

-A. ACSL4-sg2 $55 \mathrm{kDa}$

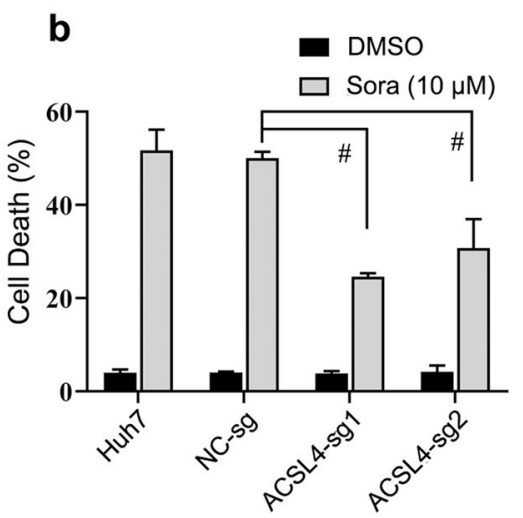

C
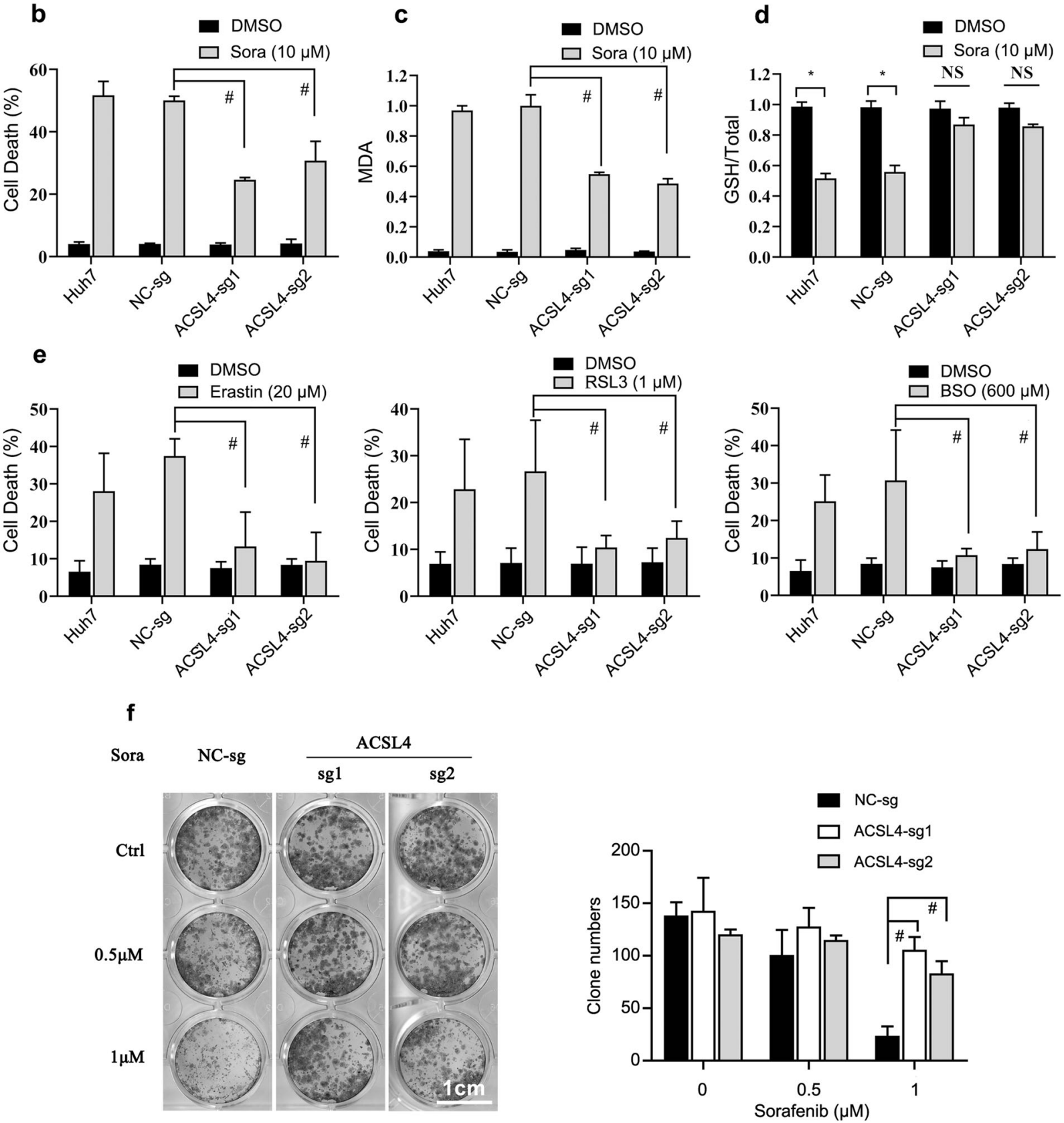

Fig. 3 Stable ACSL4-knockout Huh7 cells became resistant to ferroptosis. a Stable ACSL4-knockout Huh7 cells were generated with a CRISPR/Cas9 system. The knockout of ACSL4 was validated by Western blotting. Proliferation rates were determined with an MTT assay. $\mathbf{b}$ The role of ACSL4 in sorafenib-induced cell death was determined as described above. c, d After sorafenib was administered as in Fig. $2 c$, the changes in MDA (c) and GSH content (d) were determined. e Stable Huh7 cells were treated with another known ferroptosis inducer (20 $\mu$ M erastin, $1 \mu \mathrm{M}$ RSL3 or $600 \mu \mathrm{M}$ BSO) for $48 \mathrm{~h}$. f Stable Huh7 cells were seeded in 24-well plates and then incubated with sorafenib as indicated for 12 days before crystal violet staining. The quantification of clone numbers is shown on the right. 


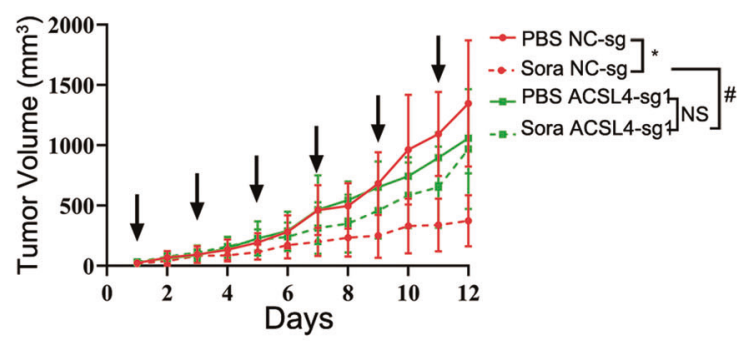

C

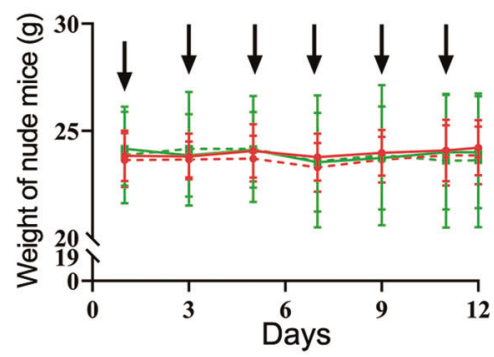

b

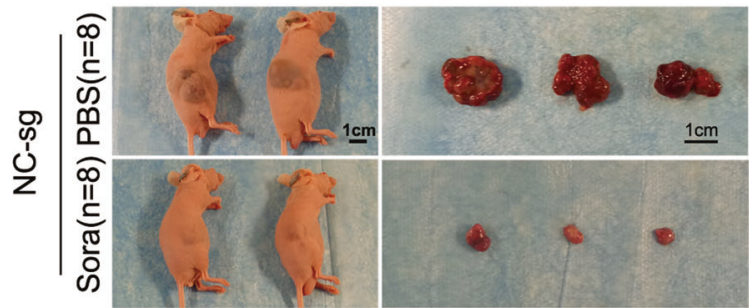

$\rightarrow$ PBS NC-sg

-. Sora NC-sg

- PBS ACSL4-sg1

-. . Sora ACSL4-sg1 d
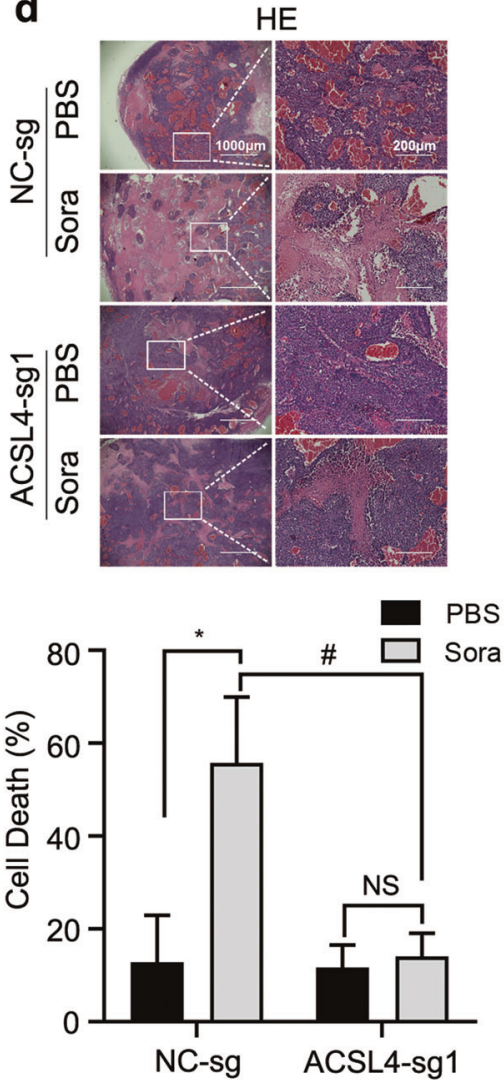
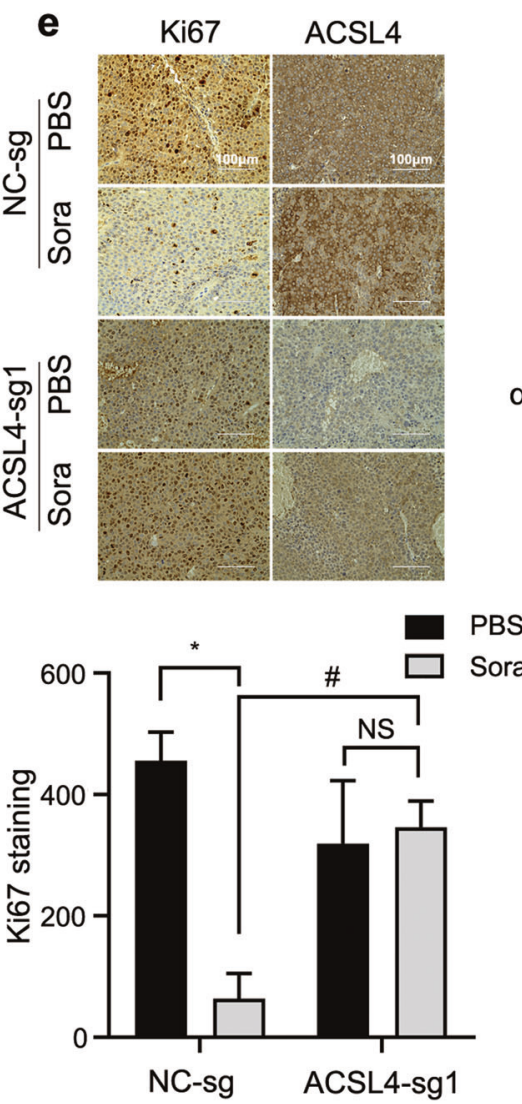

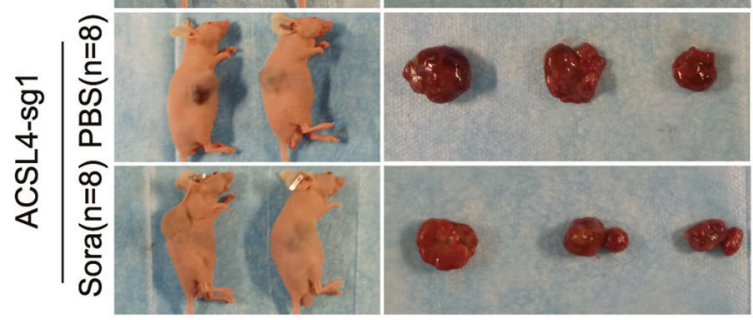

f

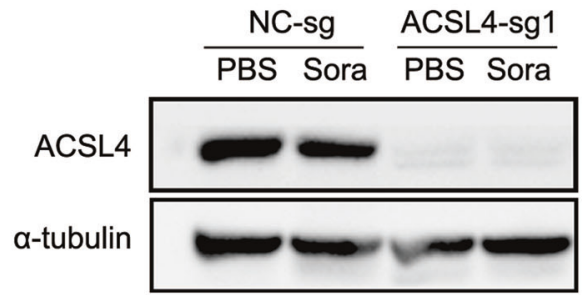

g

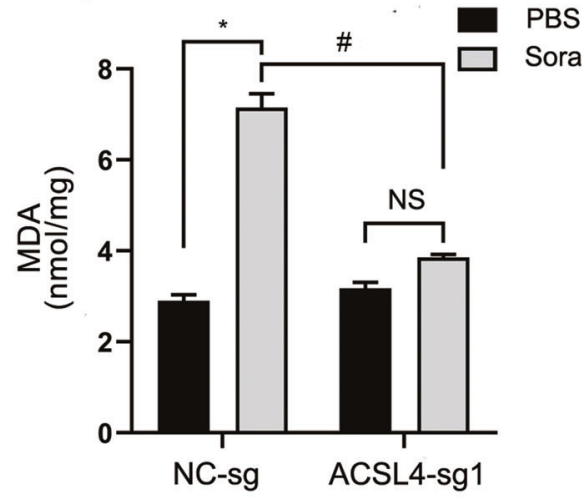

Fig. 4 ACSL4 knockout rescued sorafenib-induced xenograft growth inhibition in vivo. a Stable ACSL4-knockout cells or control cells were inoculated into the right flank of Balb/c mice ( 8 mice in each group). When the size of the generated tumor nodules reached $20-50 \mathrm{~mm}^{3}$, the mice were given sorafenib $(30 \mathrm{mg} / \mathrm{kg}$, once every other day) or the same volume of PBS by gavage. The arrow indicates sorafenib or PBS treatment. b Images of the tumor-bearing mice and resected tumor nodules were taken. The full images of the 8 mice and 8 tumor nodules in each group are shown in Supplementary Fig. 3. c The body weight of nude mice after treatment with PBS or sorafenib was measured. d Representative images of hematoxylin \& eosin staining are shown. The percentages of the total area showing cell death are summarized in the lower panel. e Representative images of IHC for Ki67 or ACSL4 are shown. Ki-67 IHC was quantified and is summarized in the lower panel. f The knockout of ACSL4 in xenograft tumors was validated by Western blotting. $\mathbf{g}$ The MDA contents in the indicated resected tumors were determined. ${ }^{*} P<0.05 ;{ }^{\#} P<0.05$.

HCC tissue was associated with a relatively good patient outcome after sorafenib treatment (Table 1). HCC patients who had high ACSL4 expression in resected tumor tissue specimens had good therapeutic outcomes after subsequent sorafenib treatment and vice versa. Thus, ACSL4 expression may act, at least partially, as a good candidate predictive biomarker for ferroptosis sensitivity in HCC. The upregulation of ACSL4 expression is not restricted to HCC. It was found that ACSL4 expression is increased in multiple 
a

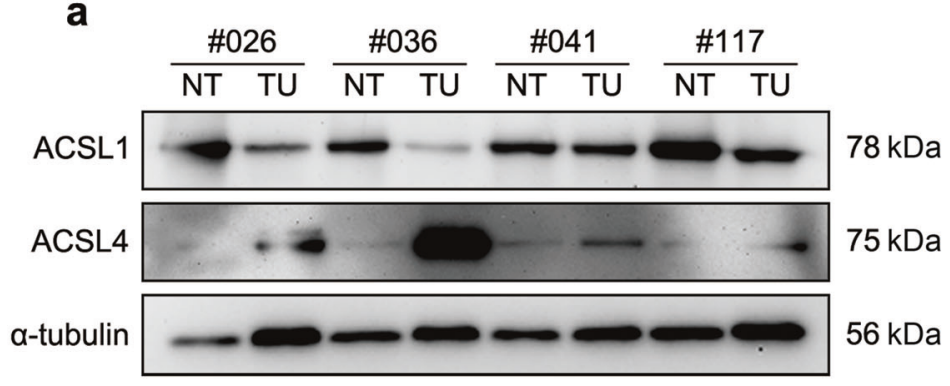

b
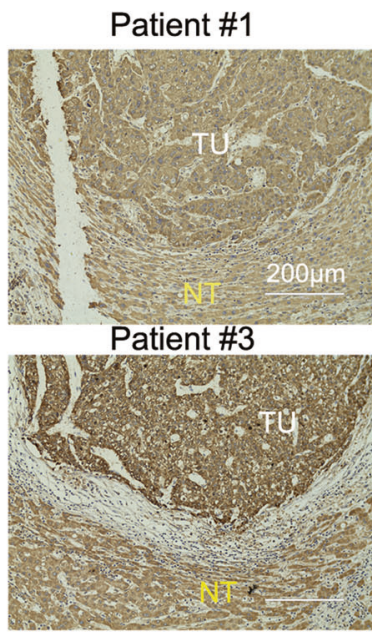

d

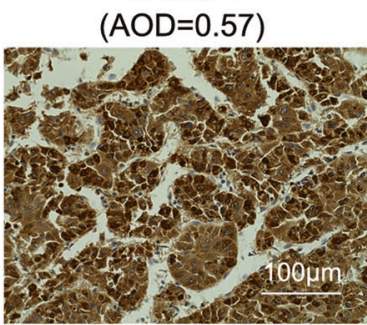

\#HCC-4

(AOD $=0.34)$

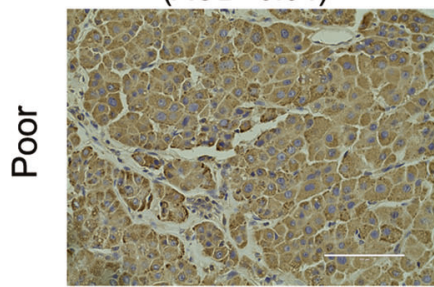

Patient \#2

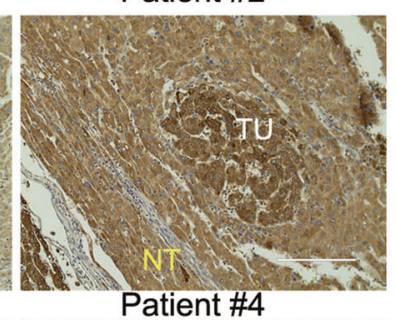

Patient \#4

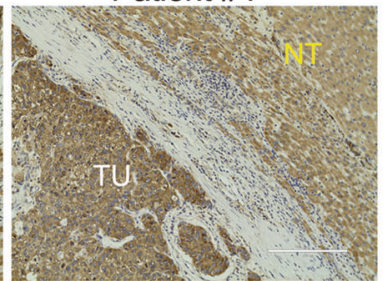

C

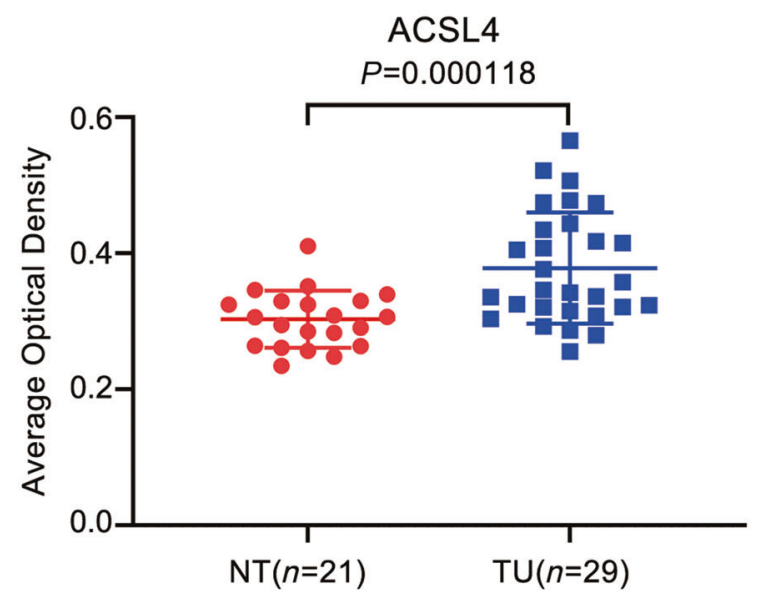

\#HCC-3

$(A O D=0.44)$

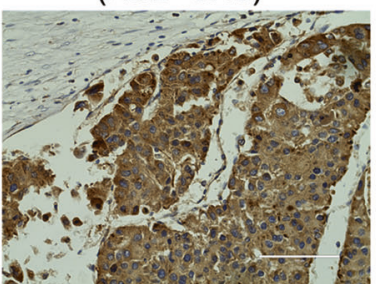

\#HCC-5

$(A O D=0.29)$

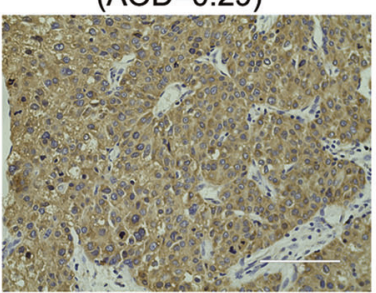

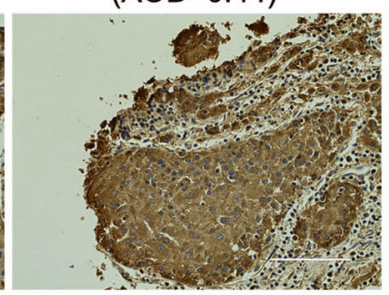

\#HCC-6

$(A O D=0.28)$

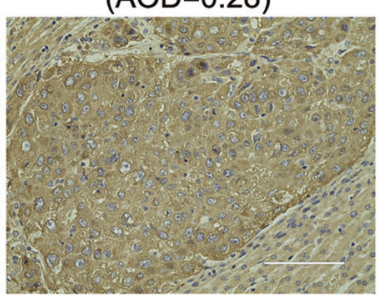

Fig. 5 ACSL4 expression was upregulated in HCC tissue specimens. a ACSL1 and ACSL4 expression was determined by Western blotting in 4 representative pairs of HCC (TU) and adjacent nontumor (NT) tissue samples. b ACSL4 protein expression was evaluated by IHC of HCC tissue sections. c ACSL4 protein expression (measured as the average optical density) in HCC (TU) and adjacent nontumor (NT) tissue samples is summarized. d Representative images of ACSL4 expression determined by IHC in samples from HCC patients with a good response or poor response are shown.

types of cancer, including liver, breast, prostate and colon cancer, but decreased in gastric cancer [30]. Thus, it is reasonable to conclude that sorafenib treatment may also work in these tumors with upregulated ACSL4 expression.

Sorafenib became the standard of care for advanced HCC in 2007. Although numerous studies have been conducted in the search for a potential biomarker to predict sorafenib sensitivity and/or HCC patient survival after sorafenib treatment during the last decade, there is currently no validated biomarker [11]. Most studies were based on changes in certain factors. For example, in sorafenib-treated advanced HCC patients, an early decrease (more than 20\%) in alpha fetoprotein (AFP) expression was associated with an improved outcome [31,32], indicating that the tumor burden was decreased by sorafenib. Side effects, particularly hand-foot skin reactions (HFSRs), were found to be consistently associated with prolonged survival in sorafenib-treated HCC patients [33-35]. These factors may identify good responders during sorafenib treatment, but their usefulness is limited in the 
Table 2. High ACSL4 expression in HCC tissue was associated with a good response in sorafenib-treated HCC patients.

\begin{tabular}{|c|c|c|c|}
\hline \multicolumn{4}{|l|}{ (a) } \\
\hline \multirow[t]{2}{*}{ ACSL4 expression in an HCC tumor (AOD) } & \multicolumn{2}{|l|}{$\begin{array}{l}\text { Response } \\
(P=0.029)\end{array}$} & \multirow[t]{2}{*}{ Total } \\
\hline & Good & Poor & \\
\hline High $(\geq 0.4)$ & $8(66.7 \%)$ & $4(23.5 \%)$ & 12 \\
\hline Low $(<0.4)$ & 4 & 13 & 17 \\
\hline Total & 12 & 17 & 29 \\
\hline \multicolumn{4}{|l|}{ (b) } \\
\hline \multirow[t]{2}{*}{ ACSL4 expression in an HCC tumor (AOD) } & \multicolumn{2}{|l|}{$\begin{array}{l}\text { Response } \\
(P=0.043)\end{array}$} & \multirow[t]{2}{*}{ Tota } \\
\hline & Good & Poor & \\
\hline High $(\geq 0.4)$ & $8(66.7 \%)$ & $2(20.0 \%)$ & 10 \\
\hline Low $(<0.4)$ & 4 & 8 & 12 \\
\hline Total & 12 & 10 & 22 \\
\hline \multicolumn{4}{|c|}{$\begin{array}{l}\text { (a) ACSL4 protein expression was determined by IHC and quantified as the } \\
\text { average optical density (AOD) with ImageJ software. The cutoff level was } \\
\text { set at AOD }=0.4 \text {, which was the } 95 \% \text { upper percentile of the ACSL4 AOD in } \\
\text { the adjacent nontumor tissue samples. This cutoff level for ACSL4 was } \\
\text { similar to that derived from the AUROC curve. As a result, ACSL4 AOD } \geq 0.4 \\
\text { was defined as high expression, while }<0.4 \text { was defined as low expression. } \\
\text { The significance of the difference was determined by Fisher's exact test } \\
\text { analysis. The number of cases involved and } P \text { values are indicated. (b) The } \\
\text { analysis was repeated in patients with a low postsurgery serological AFP } \\
\text { level }(<200 \mathrm{ng} / \mathrm{mL} \text { ). }\end{array}$} \\
\hline
\end{tabular}

prediction of sorafenib sensitivity before treatment. Inflammatory responses may reflect the cell death status in HCC tumors, angiogenesis and tumor invasion in the microenvironment. The neutrophil-to-lymphocyte ratio has been found to be an independent risk factor for survival in HCC patients treated with sorafenib [36, 37]. The molecular mechanisms involved in the NLR are currently unknown. In addition, the NLR affects patient survival in many types of solid tumors [38], suggesting its nonspecific role in sorafenib treatment. A few studies have also reported tissue biomarkers, such as phosphor-ERK [39] and c-Met [40], but many results need to be further validated.

Because of the importance of ferroptosis in sorafenib cytotoxicity to HCC cells, it is possible that proteins affecting ferroptosis may be associated with sorafenib sensitivity. Apart from a first study reporting that posttreatment changes in serum MT-1 may act as a candidate biomarker for assessing sorafenib sensitivity in HCC patients [21], our study, to the best of our knowledge, is the second to report ACSL4 as a useful biomarker to predict ferroptosis in HCC in clinical settings. In the present study, ACSL4 protein expression was found to be stable in HCC cells in vitro (Fig. $1 \mathrm{~g}, \mathrm{~h}$ ) and HCC xenografts in vivo after sorafenib treatment (Fig. $4 \mathrm{e}, \mathrm{f}$ ). This stable trait of ACSL4 is different from the changes in the expression of other redox-responsive proteins, such as GPX4 (Supplementary Fig. 1c), NRF2 [20] and MT-1 [21, 22]. These three proteins exhibited upregulated expression after the induction of ferroptosis. This stable feature of ACSL4 may enable its use as a predictive biomarker. In other words, ferroptosis sensitivity could be predicted by protein expression before sorafenib treatment rather than monitored by changes occurring after sorafenib treatment. Thus, it is feasible to predict sorafenib sensitivity or resistance in HCC patients by examining pretreatment HCC biopsies and resected tumor tissue specimens. Even for earlyrecurrent $\mathrm{HCC}$, which occurs within two years after curative surgery, examination of preserved HCC samples may still be informative. It has been suggested that the majority of earlyrecurrent HCC cases are derived from existing HCC cells in the reserved liver tissues after hepatectomy rather than from de novo carcinogenesis [3] so the gene and protein signatures of HCC are well maintained. For example, our previous study [26] showed that C/EBPalpha expression by HCC is a stable trait in tumors by examining both primary HCC tissue and early-recurrent HCC tissue. However, whether the ACSL4 expression found in primary $\mathrm{HCC}$ is applicable for prediction of recurrent HCC should be further tested in future prospective studies.

Although the basal levels of redox-responsive proteins, such as NRF2, may not be good biomarkers for evaluating subsequent sorafenib treatment outcomes, NRF2 and downstream antioxidant proteins may be good molecular targets to sensitize sorafenibinduced cell death. Genetic or pharmacological repression of NRF2 could synergize with sorafenib in inducing ferroptotic cell death in vitro and in vivo [20]. On the other hand, examination of ACSL4 may also be useful for evaluating the hepatotoxicity of sorafenib. We did observe ACSL4 expression in nontumor liver tissues (Fig. 5b), although its expression was much lower than that in the HCC tissues. This may, at least partially, explain the intolerance of sorafenib in some HCC patients. Under this circumstance, reducing the sorafenib dose or adding ACSL4 inhibitors may help alleviate unwanted side effects. ACSL4 activity can be modulated by several chemical inhibitors, e.g., rosiglitazone [28], pioglitazone [29] and 2,4thiazolidinedione [16]. All three inhibitors belong to thiazolidinediones, a class of peroxisome proliferator-activated receptor gamma (PPAR- $\gamma$ ) agonists that are used for the treatment of hyperglycemia and diabetes. Pharmacoepidemiological studies focusing on HCC patients with concurrent diabetes may help resolve whether the addition of these antidiabetic drugs affects sorafenib treatment.

Except for ACSL4, ACSL family members have not been associated with ferroptosis, to the best of our knowledge. An earlier study conducted by Yuan et al. [41] examined the associations between the mRNA levels of ACSL1, ACSL3, ACSL4, and ACSL5 (ACSL6 was not tested) and ferroptosis sensitivity in four selected cell lines, HepG2, HL60, LNCaP, and K562. Except for ACSL4, the other tested ACSL family members (ACSL1, ACSL3 and ACSL5) were not correlated with sensitivity to the ferroptosis inducer erastin. Furthermore, according to a recent paper by Magtanong et al., exogenous monounsaturated fatty acids (MUFAs) may replace plasma membrane phospholipids and then suppress ferroptosis in an ACSL3-dependent manner [42]. Therefore, ACSL4 might be specific for ferroptosis induction.

It is important to note that our clinical analysis was based on a small cohort. Unfortunately, due to the high rate of loss during follow-up, particularly among the poor-response patients, we could not perform a survival analysis. Among the poor-response patients who had an average follow-up time of $12.1 \pm 7.4$ months (median at 11 months), only two patients had clear death reports. In contrast, the good-response patients had a much greater willingness to return to hospitals for routine checkups. They had a longer follow-up time of $54.4 \pm 35.4$ months (median of 55 months and maximum of 107 months). Future studies including prospective, multicenter, randomized clinical trials are needed to validate the usefulness of ACSL4 expression as a predictive biomarker.

Overall, the present study confirmed the critical role of ACSL4 in sorafenib-induced ferroptosis in vitro and in vivo. In addition, pretreatment ACSL4 expression in resected HCC tissue can predict $\mathrm{HCC}$ tumor responsiveness to subsequent adjunct sorafenib treatment.

\section{ACKNOWLEDGEMENTS}

This work was supported by the National Natural Science Foundation of China (81672370 and 81972291), the Guangxi Natural Science Foundation Key Grant (2018GXNSFDA050006), the Key laboratory of High-Incidence-Tumor Prevention \& Treatment (Guangxi Medical University), Ministry of Education (GKE2018-02), the 
Hundred-Talent Program (2016) and the Guangxi Medical University Training Program for Distinguished Young Scholars (2017) (all awarded to GDL); and the National Natural Science Foundation of China (31601120, awarded to JZ).

\section{AUTHOR CONTRIBUTIONS}

JF and PZL equally performed the majority of the experiments and analyzed the data; GZZ collected HCC patient pathological samples and analyzed the clinical data; YW prepared the figures; XWH provided technical support; HQD, PHC, ZJL and WJS performed the literature review; $\mathrm{CYH}$ and XPY collected HCC patient clinical data; SCH provided project guidance; TP, JZ and GDL designed the research and summarized the data; and JF and GDL wrote the paper.

\section{ADDITIONAL INFORMATION}

The online version of this article (https://doi.org/10.1038/s41401-020-0439-x) contains supplementary material, which is available to authorized users.

Competing interests: The authors declare no competing interests.

\section{REFERENCES}

1. Bray F, Ferlay J, Soerjomataram I, Siegel RL, Torre LA, Jemal A. Global cancer statistics 2018: GLOBOCAN estimates of incidence and mortality worldwide for 36 cancers in 185 countries. CA Cancer J Clin. 2018;68:394-424.

2. European Association for the Study of the Liver. EASL clinical practice guidelines: management of hepatocellular carcinoma. J Hepatol. 2018;69:182-236.

3. Llovet JM, Di Bisceglie AM, Bruix J, Kramer BS, Lencioni R, Zhu AX, et al. Design and endpoints of clinical trials in hepatocellular carcinoma. J Natl Cancer Inst. 2008;100:698-711.

4. Llovet JM, Bruix J. Systematic review of randomized trials for unresectable hepatocellular carcinoma: chemoembolization improves survival. Hepatology. 2003;37:429-42.

5. Beaugrand $M$, Sala $M$, Degos F, Sherman M, Bolondi L, Evans T, et al. Treatment of advanced hepatocellular carcinoma by seocalcitol (a vit $D$ analogue): an international randomized double-blind placebo-controlled study in 747 patients. J Hepatol. 2005;42:17A.

6. Cheng AL, Kang YK, Chen Z, Tsao CJ, Qin S, Kim JS, et al. Efficacy and safety of sorafenib in patients in the Asia-Pacific region with advanced hepatocellular carcinoma: a phase III randomised, double-blind, placebo-controlled trial. Lancet Oncol. 2009;10:25-34.

7. Llovet JM, Ricci S, Mazzaferro V, Hilgard P, Gane E, Blanc JF, et al. Sorafenib in advanced hepatocellular carcinoma. N. Engl J Med. 2008;359:378-90.

8. lavarone M, Cabibbo G, Piscaglia F, Zavaglia C, Grieco A, Villa E, et al. Fieldpractice study of sorafenib therapy for hepatocellular carcinoma: a prospective multicenter study in Italy. Hepatology. 2011;54:2055-63.

9. Reig M, Rimola J, Torres F, Darnell A, Rodriguez-Lope C, Forner A, et al. Postprogression survival of patients with advanced hepatocellular carcinoma: rationale for second-line trial design. Hepatology. 2013;58:2023-31.

10. Ganten TM, Stauber RE, Schott E, Malfertheiner P, Buder R, Galle PR, et al. Sorafenib in patients with hepatocellular carcinoma-results of the observational INSIGHT study. Clin Cancer Res. 2017;23:5720-8.

11. Marisi G, Cucchetti A, Ulivi P, Canale M, Cabibbo G, Solaini L, et al. Ten years of sorafenib in hepatocellular carcinoma: are there any predictive and/or prognostic markers? World J Gastroenterol. 2018;24:4152-63.

12. Louandre C, Ezzoukhry Z, Godin C, Barbare JC, Mazière JC, Chauffert B, et al. Irondependent cell death of hepatocellular carcinoma cells exposed to sorafenib. Int J Cancer. 2013;133:1732-42.

13. Stockwell BR, Friedmann Angeli JP, Bayir H, Bush Al, Conrad M, Dixon SJ, et al. Ferroptosis: a regulated cell death nexus linking metabolism, redox biology, and disease. Cell. 2017;171:273-85.

14. Gao M, Jiang X. To eat or not to eat-the metabolic flavor of ferroptosis. Curr Opin Cell Biol. 2018;51:58-64.

15. Yang WS, SriRamaratnam R, Welsch ME, Shimada K, Skouta R, Viswanathan VS, et al. Regulation of ferroptotic cancer cell death by GPX4. Cell. 2014;156:317-31.

16. Doll S, Proneth B, Tyurina YY, Panzilius E, Kobayashi S, Ingold I, et al. ACSL4 dictates ferroptosis sensitivity by shaping cellular lipid composition. Nat Chem Biol. 2017;13:91-8.

17. Kagan VE, Mao G, Qu F, Angeli JP, Doll S, Croix CS, et al. Oxidized arachidonic and adrenic PEs navigate cells to ferroptosis. Nat Chem Biol. 2017;13:81-90.

18. Dixon SJ, Patel DN, Welsch M, Skouta R, Lee ED, Hayano M, et al. Pharmacological inhibition of cystine-glutamate exchange induces endoplasmic reticulum stress and ferroptosis. Elife. 2014;3:e02523.
19. Louandre C, Marcq I, Bouhlal H, Lachaier E, Godin C, Saidak Z, et al. The retinoblastoma $(\mathrm{Rb})$ protein regulates ferroptosis induced by sorafenib in human hepatocellular carcinoma cells. Cancer Lett. 2015;356:971-7.

20. Sun X, Ou Z, Chen R, Niu X, Chen D, Kang R, et al. Activation of the p62-Keap1NRF2 pathway protects against ferroptosis in hepatocellular carcinoma cells. Hepatology. 2016;63:173-84.

21. Houessinon A, François C, Sauzay C, Louandre C, Mongelard G, Godin C, et al. Metallothionein-1 as a biomarker of altered redox metabolism in hepatocellular carcinoma cells exposed to sorafenib. Mol Cancer. 2016;15:38.

22. Sun X, Niu X, Chen R, He W, Chen D, Kang R, et al. Metallothionein-1G facilitates sorafenib resistance through inhibition of ferroptosis. Hepatology. 2016;64:488-500.

23. Eisenhauer EA, Therasse P, Bogaerts J, Schwartz LH, Sargent D, Ford R, et al. New response evaluation criteria in solid tumours: revised RECIST guideline (version 1.1). Eur J Cancer. 2009;45:228-47.

24. Lu GD, Leung CH, Yan B, Tan CM, Low SY, Aung MO, et al. C/EBPalpha is upregulated in a subset of hepatocellular carcinomas and plays a role in cell growth and proliferation. Gastroenterology. 2010;139:632-43.

25. Zhou J, Tan SH, Valérie N, Bauvy C, Yang ND, Zhang J, et al. Activation of lysosomal function in the course of autophagy via mTORC1 suppression and autophagosome-lysosome fusion. Cell Res. 2013;23:508-23.

26. Lu GD, Ang YH, Zhou J, Tamilarasi J, Yan B, Lim YC, et al. CCAAT/enhancer binding protein a predicts poorer prognosis and prevents energy starvation-induced cell death in hepatocellular carcinoma. Hepatology. 2015;61:965-78.

27. Schneider CA, Rasband WS, Eliceiri KW. NIH image to ImageJ: 25 years of image analysis. Nat Methods. 2012;9:671-5.

28. Askari B, Kanter JE, Sherrid AM, Golej DL, Bender AT, Liu J, et al. Rosiglitazone inhibits acyl-CoA synthetase activity and fatty acid partitioning to diacylglycerol and triacylglycerol via a peroxisome proliferator-activated receptor-gammaindependent mechanism in human arterial smooth muscle cells and macrophages. Diabetes. 2007;56:1143-52.

29. Heneka MT, Fink A, Doblhammer G. Effect of pioglitazone medication on the incidence of dementia. Ann Neurol. 2015;78:284-94.

30. Tang Y, Zhou J, Hooi SC, Jiang YM, Lu GD. Fatty acid activation in carcinogenesis and cancer development: essential roles of long-chain acyl-CoA synthetases. Oncol Lett. 2018;16:1390-6.

31. Shao YY, Lin ZZ, Hsu C, Shen YC, Hsu CH, Cheng AL. Early alpha-fetoprotein response predicts treatment efficacy of antiangiogenic systemic therapy in patients with advanced hepatocellular carcinoma. Cancer. 2010;116:4590-6.

32. Personeni N, Bozzarelli S, Pressiani T, Rimassa L, Tronconi MC, Sclafani F, et al. Usefulness of alpha-fetoprotein response in patients treated with sorafenib for advanced hepatocellular carcinoma. J Hepatol. 2012;57:101-7.

33. Reig M, Torres F, Rodriguez-Lope C, Forner A, Llarch N, Rimola J, et al. Early dermatologic adverse events predict better outcome in HCC patients treated with sorafenib. J Hepatol. 2014;61:318-24.

34. Wang P, Tan G, Zhu M, Li W, Zhai B, Sun X. Hand-foot skin reaction is a beneficial indicator of sorafenib therapy for patients with hepatocellular carcinoma: a systemic review and meta-analysis. Expert Rev Gastroenterol Hepatol. 2018;12:1-8.

35. Abdel-Rahman O, Lamarca A. Development of sorafenib-related side effects in patients diagnosed with advanced hepatocellular carcinoma treated with sorafenib: a systematic-review and meta-analysis of the impact on survival. Expert Rev Gastroenterol Hepatol. 2017;11:75-83.

36. Bruix J, Cheng AL, Meinhardt G, Nakajima K, De Sanctis Y, Llovet J. Prognostic factors and predictors of sorafenib benefit in patients with hepatocellular carcinoma: analysis of two phase III studies. J Hepatol. 2017;67:999-1008.

37. da Fonseca LG, Barroso-Sousa R, Bento Ada S, Blanco BP, Valente GL, Pfiffer TE, et al. Pre-treatment neutrophil-to-lymphocyte ratio affects survival in patients with advanced hepatocellular carcinoma treated with sorafenib. Med Oncol. 2014;31:264

38. Templeton AJ, McNamara MG, Šeruga B, Vera-Badillo FE, Aneja P, Ocaña A, et al. Prognostic role of neutrophil-to-lymphocyte ratio in solid tumors: a systematic review and meta-analysis. J Natl Cancer Inst. 2014;106:dju124.

39. Personeni N, Rimassa L, Pressiani T, Destro A, Ligorio C, Tronconi MC, et al. Molecular determinants of outcome in sorafenib-treated patients with hepatocellular carcinoma. J Cancer Res Clin Oncol. 2013;139:1179-87.

40. Chu JS, Ge FJ, Zhang B, Wang Y, Silvestris N, Liu LJ, et al. Expression and prognostic value of VEGFR-2, PDGFR- $\beta$, and c-Met in advanced hepatocellular carcinoma. J Exp Clin Cancer Res. 2013;32:16.

41. Yuan H, Li X, Zhang X, Kang R, Tang D. Identification of ACSL4 as a biomarker and contributor of ferroptosis. Biochem Biophys Res Commun. 2016;478:1338-43.

42. Magtanong L, Ko PJ, To M, Cao JY, Forcina GC, Tarangelo A, et al. Exogenous monounsaturated fatty acids promote a ferroptosis-resistant cell state. Cell Chem Biol. 2019;26:420-32. 